\title{
Sonderausstellung zur Jenner-Feier im Medicinischen Waarenhause zu Berlin
}

Zur hundertjährigen Ehrung der Schutzpocken - Jmpfuug hat das Berliner Jenner-Comité im Medicinischen Waarenhause eine höchst be-merkenswerte Ausstellung veranstaltet. Dieselbe umfasste in ihrem knappen, übersichtlichen Rahmen alle Cfebiete des Impfwesens und giebt ein litte-rarisch-historisches Bild von einleuchtender Klarheit. Auch alle techniseh im VaccinationsSaeculum gewonnenen Fortschritte und wissenschaftlichen Errungenschaften kamen dahei zur Anschauung. Dankbar bezeugen dies zahlreiche Besucher aus ärztlichen und studentischen Kreisen. Das Haupt-Verdienst der Einrichtung und Leitung dieser intoressanten Ausstellung ist unserm verehrten Collegen, Herrn Gustav Behrend, zuzuschreiben, der dieselbe in Gl·egenwart einer glänzenden Versammlung mit folgender Rede1) eröffnete:

Hochverehrte Versammlung!

Dme Comité íiir die Hundertjahresfeier der Entdeckung der Vaccination gereicht es zur besonderen Ehre, Sie in diesen Riiumen begrüssen zu können. Nicht in prunkendem Schmuck und durch Darstellungen, welche das Auge fesseln, nicht mit Sang und Klang, welche das Ohr ergötzen oder das Herz erfreuen, trete $\pi$ wir in die Oeffentlichkeit, ein-fach und in häuslichem Gewande, still und bescheiden, wie es Art der Wissenschaft ist, wie es war und auch íernerhin seiu wird, wollen wir die wichtigsten Marksteine in der Greschichte der Vaccination vorführea. Sprechende Zeugen aus vergangener Zeit sollen erzählen, wie sie in stürmendem Siegeslauf in kaum einem Decennium die Welt erohert, sprechende Zeugen sollen`darthun, wie in sieben Jahrzehnten des ver-

1) Nach dem Stenogramm der Deutschen Aerzte-Zeitung No. 10. 1896.

408 Mitt $\beta$ ilung $\beta$ n.

gangenen Jahrhunderts der Boden vorbereitet wurde, auf welchem die Frucht zur Entwickelung gelangte. Segen ist der Menschheit zuteil ge-worden, so hörte man von den Kanzeln, so lehrte man in den Schulen, sobald man erfahren hatte, dass jener Peind besiegbar sei, der in kurzen Intervallen stets wiederkehrend das Menschengeschlecht decimierte. , Die ausgestellten Schriften, welche nur einen Bruehteil derer bilden, die bis etwa ' zum 2. Decennium dieses Jabrbunderts über die Vaccination er-schienen sind, zeugen nocb heute davon, dass sich das Menscbengeschlecht von einem drückenden Alp befreit fühlte.

Was aber die Vaccination im Völkerleben bedeutet, davon baben Fürsten aller Lander ein beredtes Zeugnis abgelegt, indem sie Denk-münzen prägen liessen zur dauernden Erinnerung an den ihren Kindern erteüten Impfscbutz, wertvolle Denkmünzen aucb für Aerzte, welche durch die That und für Geistliche, welche durch das Wort bei der Aus-breitung jener segensreichen Entdeckung wirksame Hilfe liehen. Fast alle diese Münzen sind zur Stelle, um selber Zeugnis abzulegen.

Und wie die Impfung, wie die Darstellung und Conservierung des Impfstoffes im Laufe der Jahre Gegenstand unausgesetzter Bemíihungen waren, haben wir in einer weiteren Gruppe von Objecten zur Anschauung gebracht. Die complicierten Instrumente, welche man erfand, sind 
längst vergessen; mit der Antisepsis und mit der Verwendung animalen Impf-stofi $1 / 8$, deren Darstellung wir gleichfalls vor Augen fübren, sind alle jene Mangel beseitigt, die der Vaccination früher zur Last fielen.

So blicken denn die Manen jener Manner, deren Bildnisse heute diesem Raum zum höchsten Schmuck gereichen, gleichsam herab auf das Werk, welches sie geschaffen, und das von nachwachsenden Geschlechtern ausgebaut und gefördert wurde. Und wir, die Enkel, werden jenes kost-bare Vermächtnis, das schon längst zum Eigentum der Menschheit wurde, schützen. schützen mit den Waffen, die es selbst geschmiedet in unsere Hand gelegt: und diese Waffen heissen „Wissenschaft” und „Wahrheit”. Und deshalb treten wir auch unseren Gegnern mit freier Stirn und offenem Ange gegenüber, deshalb haben wir auf einmütigen Beschluss des Comités auch ihnen einen Platz neben uns angewiesen. Ueber die Ausbreitung der Pocken in Enropa im vorigen Jabrhundert und über den Einfluss, welchen die Vaccination auf die Pockensterblichkeit ausgeübt hat, davon legen die Tafeln, welche wir dem Reichsgesundheitsamte verdanken, ein beredtes Zeugnis ab.

Dass uns ermöglicbt wurde das herbeizuschaffen, was wir bieten, verdanken wir dem freundlichen Entgegenkomnien der Behörden und Privater. Durch das freundliche Entgegenkom nen Sr. Excellenz des Herrn Generalstabsarzt der Armee Prof. Dr. v. Coler wurden uns die Sammlung der Kaiser-Wilhems-Akademie durch den Director des hy-gieinischen Instituts, Herrn Prof. Rubner, die Sammlung seines Museums zur Verfügung gestellt. Die Köngl. LympheErzeugungsanstalt zu Berlin hat wertvolle Beiträge geliefert, und die Anstalten für animale Vaccine in Bremen, Bautzen, Rom, Buenos Ayres Madras, sowie das Impfinstitut von Dr. Pissin in Berlin weitere wertvolle Beiträge, Herr Medicinal-rath Dr. Pfeif fer in Weimar hat wertvolle Tafeln, die sich auf Pocken und Kuhpocken beziehen und seine vollständige Medaillensammlung ein-gesandt, Herr Apotheker Runge in Berlin, Dr. Renner in London

Mitt $\theta$ ilung $\cdot e n$.

409

reiche Beiträge zur Erinnerung an Jenner (Bilder und Briefe), Herr Assessor Pohlandt in Berlin wertvolle Familienstücke zur Erinnerung an Bremer, den ersten Impfarzt in Berlin, der Magistrat in Bückeburg die Gegenstäßde, welche zu dem alljährlich stattfindenden Kinder-Jubelfest zur Entdeckung der Vaccination daselbst siïftungsmässig zur Aus-schmückung des Platzes im Walde dienen. Diesen und alien andern Gönnern unseres Unternehmens gebührt der Dank des Comité $1 / 8$ !

Und so eröffne ich diese Ausstellung im Namen der Wissenschaft und der Wahrheit und im Auftrage des Comité's für die Hundertjahres-feier der Entdeckung Eduard -Tenner's unter der Devise: Salus hominum, medicorum suprema lex.

Für den III. internationalen Dermatologen - Kongress, welcher in London vom 4. bis 8. August stattfmdet, wurde folgendes Programm ausgegeben. Dienstag: 4. August, 11 Uhr Vorm.: Vorbereitende Geschäfts-sitzung. - 12 Uhr Vorm.: Eröffnungsrede des Präsidenten. - 3 Uhr Nachm. Sitzung: „Ueber Prurigo.” 1. Dr. Besnier (Paris). 2. Prof. Kaposi (Wien). 3. Dr. J. C. White (Boston). 4. Dr. Payne (London).

Mittwoch, 5. August, 9 Uhr Vorm.: Klinische Demonstrationen.

- $\quad$ Dermatologie. IOY2 Uhr Vorm.: Aetiologie und Pormen der

Keratosen. 1. Dr. Unna (Hamburg). 2. Dr. H. G. Brooke (Man

chester). 3. Prof. V. Mibelli (Parma). 4. Dr. W. Dubreuilh

(Bordeaux). - Syphilis. 1O1^ Uhr Vorm.: Reinfectio syphilitica.

1. Prof. Fournier (Paris). 2. Prof. Lang (Wien). 3. Alfred Cooper 
(London). 4. Dr. Fitzgibbon (Dublin). 2 Uhr Nachm.: Klinische

Demonstrationen. - 3 Uhr Nachm.: Vorträge.

Donnerstag: 6. August. 9 Uhr Vorm.: Klinische Demonstrationen. Derm. 1O1/^ Uhr Vorm.:

Beziehungen der Tuberkulose zu Haut-erkrankungen mit Anschluss des Lupus vulgaris. 1. Dr. J. Nevins Hyde (Chicago). 2. Dr. Hallopeau (Paris). 3. Dr. Radcliffe Crocker (London). 4. Prof. G. Riehl (Leipzig). - Syph. 10y2 Uhr Vorm.: Dauer der Uebertragbarkeit der Syphilis. 2.

Hutchinson (London). 2. Prof. Campana (Rom). 3. Prof. Lassar (Berlin). 4. Dr. Feulard (Paris).

- 2 Uhr Nachm.: „Herpes tonsurans und Trichophytonarten.” 1. Dr.

Sab our and (Paris). 1. Prof. Rosenbach (Göttingen). 3. Malcolm

Morris (London). Zur Debatte vorgemerkt: Dr. Unna, Dr. Colcott

Fox. Leslie Roberts u. A.

410

Mitteilungen.

Preitag, 7. August, 9 Uhr Vorm.: Klinische Demonstrationen. - Derm. 1O1^ Uhr Vorm.: Natur und Beziehungen der verschiedenen Formen des Erythema multiforme. J. Prof, de Amicis (Neapel). 2. Dr. T. H. Veiel (Stuttgart). 3. Dr. Prince A. Morrow (New-York). 4. Stephen Mackenzie (London). - Syph. 1O1^ Uhr Vorm.: Maligne Syphilis. 1. Prof. Tarnovsky (St. Petersburg). - 2 Uhr Nacbm.: Klinische Demonstrationen- - 3 Uhr Vorträge.

Samstag, 8. August, 3 Uhr: Klinische Demonstrationen und Vorträge.

Alle Herren, welche Mitglieder des Kongresses zu werden wünschen, wollen sich möglichst bald entweder mit dem Generalsekretär Herrn Dr. Pringle oder mit dem Sekretär für Deutschland Herrn Dr. 0. Rosen-thai, Berlin W., Potsdamerstrasse 23, in Verbindung setzen, an welch1 Letzteren auch der Mitgliedsbeitrag von 1 Pfd. Sterling entrichtet werIen kann. Auskünfte über Wohnungen u. dgl. ertheilt Herr Dr. Pringle 23 Lower Seymour Street, Portman Square, London W. 